UCID- 21755

\author{
THE EFFECT OF PRECIPITATION CONDITIONS \\ AND AGING UPON CHARACTERISTICS OF \\ PARTICLES PRECIPITATED FROM AQUEOUS SOLUTIONS
}

Joseph A. Rard

October, 1989

This is an informal report intended primarily for internal or limited external distribution. The opinions and conclusions stated are those of the author and may or may not be those of the Laboratory.

Work performed under the auspices of the U.S. Department of Energy by the Lawrence Livermore National Laboratory under Contract W-7405-Eng-48. 


\section{DISCLAIMER}

This document was prepared as an account of work sponsored by an agency of the United States Government. Neither the United States Government nor the University of California nor any of their employees, makes any warranty, express or implied, or assumes any legal liability or responsibility for the accuracy, completeness, or usefulness of any information, apparatus, product, or process disclosed, or represents that its use would not infringe privately owned rights. Reference herein to any specific commercial products, process, or service by trade name, trademark, manufacturer, or otherwise, does not necessarily constitute or imply its endorsement, recommendation, or favoring by the United States Government or the University of California. The views and opinions of authors expressed herein do not necessarily state or reflect those of the United States Government or the University of California, and shall not be used for advertising or product endorsement purposes.

This report has been reproduced

directly from the best available copy.

Available to DOE and DOE contractors from the

Office of Scientific and Technical Information

P.O. Box 62, Oak Ridge, TN 37831

Prices available from (615) 576-8401, FTS 626-8401.

Available to the public from the

National Technical Information Service

U.S. Department of Commerce

5285 Port Royal Rd.,

Springfield, VA 22161

Papercopy Prices 


\section{DISCLAIMER}

This report was prepared as an account of work sponsored by an agency of the United States Government. Neither the United States Government nor any agency Thereof, nor any of their employees, makes any warranty, express or implied, or assumes any legal liability or responsibility for the accuracy, completeness, or usefulness of any information, apparatus, product, or process disclosed, or represents that its use would not infringe privately owned rights. Reference herein to any specific commercial product, process, or service by trade name, trademark, manufacturer, or otherwise does not necessarily constitute or imply its endorsement, recommendation, or favoring by the United States Government or any agency thereof. The views and opinions of authors expressed herein do not necessarily state or reflect those of the United States Government or any agency thereof. 


\section{DISCLAIMER}

Portions of this document may be illegible in electronic image products. Images are produced from the best available original document. 


\title{
The Effect of Precipitation Conditions and Aging Upon Characteristics of Particles Precipitated from Aqueous Solutions
}

\author{
Joseph A. Rard \\ Earth Sciences Department \\ Lawrence Livermore National Laboratory
}

\section{DISCLAIMER}

This report was prepared as an account of work sponsored by an agency of the United States Government. Neither the United States Government nor any agency thereof, nor any of their employees, makes any warranty, express or implied, or assumes any legal liability or responsibility for the accuracy, completeness, or usefulness of any information, apparatus, product, or process disclosed, or represents that its use would not infringe privately owned rights. Reference herein to any specific commercial product, process, or service by trade name, trademark, manufacturer, or otherwise does not necessarily constitute or imply its endorsement, recommendation, or favoring by the United States Government or any agency thereof. The views and opinions of authors expressed herein do not necessarily state or reflect those of the United States Government or any agency thereof. 


\begin{abstract}
Precipitation of a dissolved species from aqueous solutions is one of the techniques used to grow particles with certain size or composition characteristics. Various factors affecting the particle properties for sparingly soluble substances are briefly discussed here, including homogeneous versus heterogeneous nucleation, the effect of relative supersaturation on the number of nuclei and their relative size, particle growth by way of Ostwald Ripening, the Ostwald Step Rule and nucleation of metastable phases, diffusioncontrolled versus surface reaction-controlled growth, incorporation of dopants into the precipitate, and dendritic growth.
\end{abstract}

\title{
1. General Considerations
}

A solution is defined to be supersaturated when it has a concentration higher than the equilibrium saturation value for a particular solid phase that might be expected to form. Supersaturation can be achieved by several different methods:

1. By evaporating part or all of a solvent under isothermal or nearly isothermal conditions. Examples of this include evaporation in a desiccator and evaporation of solvent with a vacuum or partial vacuum.

2. By evaporating solvent due to heating the solution. This will sometimes produce different solid phases than evaporation at room temperature.

3. By cooling the sample to reduce the solubility of a solute. Very rapid cooling tends to produce glassy phases rather than crystalline solids. In a few cases (e.g., hydrated rare earth sulfates), supersaturation is achieved by heating the solutions instead, since they exhibit retrograde solubility.

4. By mixing together solutions of soluble solutes that then react to form a much less soluble compound or salt.

5. By adding a second solvent or another solute to the solution to reduce the solubility of a dissolved solute. Reduction of the solubility of a solute by addition of one or more salts is known as "salting out."

Supersaturated solutions are metastable with regard to precipitation of a solid phase. There is a metastable supersaturated concentration region for any given solute in a specific solvent at a fixed temperature. In this metastable supersaturation region, solutions can appear to be "stable" for extended periods of time. However, if a nucleation site is present, then crystallization of the solute will occur. In addition, as the concentration of the supersaturated solute is increased further in the absence of a nucleation site, a point in the 
unstable supersaturated region will ultimately be reached. Here solutions are completely unstable and will spontaneously nucleate.

The number, size, and chemical characteristics of these precipitating particles will depend on precipitation conditions, especially the degree of supersaturation. If these particles are allowed to remain in contact with the mother liquor, smaller particles are generally observed to shrink in size and ultimately disappear; in contrast, the larger particles grow with time. This is true for any particular chemical type of particle. If a metastable solid phase forms initially, then it will tend to transform into a more stable phase. Examples include an amorphous solid transforming into a crystalline phase and changes in the stoichiometry of a solid phase. This can be extremely slow in some cases, especially when the thermodynamically stable form of the solid differs significantly from the starting material in terms of structure and Gibbs energy.

The various factors briefly mentioned in this section will now be considered in more detail.

\section{Gibbs Energies and Affinity}

Consider the simple case of one solute dissolved in one solvent. The Gibbs energy change for a phase change is given by

$\Delta \mathrm{G}=\mathrm{G}_{2}-\mathrm{G}_{1}$

where 2 denotes the solute in a solid phase (precipitate) and 1 denotes the solute in the dissolved phase (solution). The chemical potential $\mu_{i}$ is given by $G_{i} / n_{i}$ for pure phases, that is $G_{i}$ per mole of $i$, and $\partial G / \partial n_{i}$ for components of a solution phase. Thus,

$\Delta \mu=\mu_{2}-\mu_{1}=-\underline{\mathrm{A}}$

where $\underline{A}$ is the affinity. For any chemical reaction in general,

$\underline{\mathrm{A}}=-\Sigma_{\mathrm{i}} \mathrm{v}_{\mathrm{i}} \mu_{\mathrm{i}}$ (products) $+\Sigma_{\mathrm{i}} \mathrm{v}_{\mathrm{i}} \mu_{\mathrm{i}}$ (reactants)

where the $v_{\mathrm{i}}$ are stoichiometric reaction coefficients. For any equilibrium situation, $\Delta \mathrm{G}=0$. Thus

$\Sigma_{\mathrm{i}} v_{\mathrm{i}} \mu_{\mathrm{i}}$ (products) $-\Sigma_{\mathrm{i}} v_{\mathrm{i}} \mu_{\mathrm{i}}$ (reactants) $=0$

and $\underline{\mathrm{A}}=0$ at equilibrium. 
The chemical potential for a substance in a mixture is given by

$\mu_{1}=\mu_{1}^{o}+R T \ln a_{1}$

where $a_{1}$ is the activity of that substance in a particular phase. For a pure precipitate phase $a_{1}=a_{1}^{o}=1$, so $\mu_{1}=\mu_{1}^{o}$.

These equations for solid phases are valid if surface energy contributions to the Gibbs energy are negligible compared to the Gibbs energy of the bulk phase. If the precipitate is finely dispersed, as it is when small nuclei are forming in the initial stages of crystallization, then the surface Gibbs energy can be a significant part of the total. This is discussed in Sections 3 and 4.

If we now consider a precipitated solid in equilibrium with its saturated solution, then $\underline{A}_{s}=0$ and

$\mu_{1 s}=\mu_{1}^{o}+R T \ln a_{1 s}$.

Here $a_{1 s}$ is the activity of the dissolved solute in its saturated solution. For a solution whose concentration differs from the saturation value,

$\underline{A}=-\left(\mu_{1}-\mu_{1 s}\right)=-R T \ln \left(a_{1} / a_{1 s}\right)$

where $\left(a_{1} / a_{1 s}\right)$ is sometimes called the saturation index. If we consider a hydrated solid that dissociates into ions upon dissolution, then

$\mathrm{M}_{\mathrm{x}} \mathrm{A}_{\mathrm{y}} \cdot \mathrm{nH} \mathrm{H}_{2} \mathrm{O}(\mathrm{s})=\mathrm{xM}^{+\mathrm{y}}($ dissolved $)+\mathrm{yA}^{-\mathrm{x}}$ (dissolved) $+\mathrm{nH}_{2} \mathrm{O}$ (solution)

This dissolved solute will have an activity given by

$a_{1}=x^{x} y \gamma_{M}^{y} \gamma_{A}^{y} C^{x+y} a_{H_{2}} O^{n}$

where $C$ is the concentration of dissolved solute on some yet-unspecified concentration scale. If we now restrict this discussion to a sparingly soluble solute, then $\mathrm{a}_{\mathrm{H}_{2} \mathrm{O}}$ will be close to the pure water value of 1 . In addition, the activity coefficients $\gamma_{M}$ and $\gamma_{A}$ will not deviate much from unity under this condition. Thus,

$a_{i} \approx x^{x} y^{y} C^{x+y}$.

Then, for a solute not at saturation, Equation (7) becomes

$\underline{A}=-R T \ln \left(x^{x} y^{y} C^{x+y} / x^{x} y^{y} C_{s}^{x+y}\right)=-(x+y) R T \ln \left(C / C_{s}\right)$. 
If we now consider an unsaturated solution, $C_{u} / C_{s}<1$ and $\ln \left(C_{u} / C_{s}\right)$ is negative. Thus $\underline{A}>0$ and $\Delta G<0$. Since we know that $\Delta G$ is negative for a spontaneous process, this agrees with the obvious fact that dilution of a saturated solution produces a stable unsaturated solution.

Instead, consider an oversaturated solution (supersaturated solution). Here $C_{o} / C_{s}>1$ and $\ln \left(C_{o} / C_{s}\right)$ is positive. Thus $\underline{A}<0$ and $\Delta G>0$. For any process with $\Delta G>0$, we know an unstable situation is produced, and something must change so that $\Delta \mathrm{G} \leq 0$. In our case, the obvious change will be crystallization. However, as we have noted already, oversaturated solutions can be metastable for long periods of time without any crystallization occurring. The case of spontaneous homogeneous nucleation is considered in the next section.

\section{Spontaneous Homogeneous Nucleation}

Nucleation theory is based on the assumption that any homogeneous single phase will undergo spontaneous fluctuations in density or concentration (Nielsen, 1964). They should occur much more rapidly for the fluid phases (liquid and gas) and over a much longer time scale in solids and glasses owing to their higher viscosities. These are known as homophase fluctuations and they continually form and disappear. Occasionally these variations will be large enough to have some characteristics of a different phase (e.g., critical opalescence); they are then known as heterophase fluctuations and can ultimately give rise to formation of a second phase by precipitation. Nucleation theory invokes the assumption that these heterophase fluctuations grow by addition of single molecules or ions to the clusters. If these heterophase fluctuations become large enough to initiate formation of a second phase, then we shall call them embryonic nuclei.

Fluctuations in concentration or density always involve changes in Gibbs energy. If these clusters are below the critical size for particle growth (see below), then equilibrium between the parent phase and the fluctuations can be assumed. We can then assume the number of clusters is given by a Boltzmann distribution,

$N_{i}=N_{i}^{0} \exp \left(-\Delta G_{i}^{*} / R T\right)$

where $\mathrm{N}_{\mathrm{i}}$ is the number of clusters per unit volume, $\mathrm{N}_{\mathrm{i}}^{0}$ is the number of molecules (or number of $M_{x} A_{y}$ that can form from individual ions, which we now term a monomer unit) per unit volume of the reactant phase, and $\Delta \mathrm{G}_{i}^{*}$ is the Gibbs energy of a cluster containing $i$ monomeric units.

If the solution is within its region of thermodynamic stability (unsaturated), then the probability of forming clusters decreases rapidly with size of the cluster, and no cluster can reach the critical size for unrestrained growth. Thus, no heterophase fluctuation is 
thermodynamically stable, and no phase separation will occur. This is in agreement with the obvious situation that unsaturated solutions will not precipitate.

If the solution phase is outside its region of stability (i.e., is supersaturated), then the Gibbs energy of a heterophase can be lower than that of the initially homogeneous solution phase. The Gibbs energy as we have been using it in the above discussion is a bulk phase value. That is, we assumed that the sample is large enough that surface energy contributions are negligible compared to the Gibbs energy of the bulk phase.

In a bulk phase, any atom or molecule (or monomer) will be surrounded on all sides by others of the same type, and they will be equivalent to each other in a thermodynamic sense. However, those monomers on the surface of this bulk phase will be surrounded by identical monomers only in certain directions; on the other sides, its environment will involve a different phase and composition. Chemical forces will thus be different in the bulk and surface phases.

It is likely that the surface energy of a particle per unit area is shape dependent; i.e., it will depend on curvature. However, since surface energies of solids are generally poorly characterized experimentally, it will not hurt this discussion to assume a constant average surface value for a unit area of surface no matter what the size or shape of the particle is. It should be noted that the surface energy of a substance will depend on the liquid or gas it is in contact with. For example, the surface energy of $\mathrm{CaCO}_{3}$ will differ slightly when it is in contact with water, ethyl alcohol, or air.

The total Gibbs energy change for the formation of a cluster of $j$ monomer units is

$\Delta G_{t}=j \Delta g_{i}+j^{2 / 3} \sigma \delta$

where $\Delta \mathrm{g}_{\mathrm{i}}$ is now the chemical part of the Gibbs energy of a monomer unit, $\delta$ is the surface energy per unit surface area, and $\sigma$ is a geometric shape factor. The total surface energy will also be given by $\delta \times A$, where $A$ is the surface area of the cluster. We wrote $A=j^{2 / 3} \sigma$ to explicitly look at the dependence of $\Delta G_{t}$ on cluster size.

Now let us consider some simple geometries. For a single spherical particle, $A=4 \pi r^{2}$ and

$\Delta G_{t}=(4 / 3) \pi r^{3} \Delta G_{v}+4 \pi r^{2} \delta$

where $\Delta G_{v}$ is now the chemical part of the Gibbs energy change per unit volume of cluster formed. For large radii, the first term dominates and in this limit the Gibbs energy just becomes that for the pure solid cluster (and it is negative). However, for small radii, the clusters will have a very large surface area per unit volume and the surface energy term (a 
positive value) will dominate $\Delta G_{t}$. Thus, as the radius is increased from very small values to larger values, $\Delta G_{t}$ first increases, goes through a maximum, and then decreases.

The point at which $\Delta \mathrm{G}_{\mathrm{t}}$ has a maximum is given by

$\mathrm{d} \Delta \mathrm{G}_{\mathrm{t}} / \mathrm{dr}=0$.

Differentiating Equation (14) then gives

$4 \pi r_{c}^{2} \Delta G_{v}+8 \pi r_{c} \delta=0$

where $r_{c}$ is the critical radius at which this occurs, and thus

$r_{c}=-\left(2 \delta / \Delta G_{v}\right)$

We have implicitly assumed in this derivation that we are not near a critical solution point.

What is $r_{c}$ ? It is the radius at which $\Delta G_{t}$ changes from an increasing to a decreasing function of particle size. That is, clusters that form with $r<r_{c}$ will spontaneously redissolve in order to reduce the total Gibbs energy. However, particles with $r>r_{c}$ will decrease the total Gibbs energy by growing. Thus $r_{c}$ is the critical radius; above this value, cluster growth and thus nucleation will be spontaneous. The "equilibrium" concentration of critical nuclei is then given by

$N_{c}=N_{i} \exp \left(-\Delta G_{t} / R T\right)$.

The above derivation oversimplifies the actual physical situation. There is usually an activitation barrier that must be overcome for attachment of a molecule or ion to occur.

Thus,

$N_{c} \propto \exp \left(-\Delta G_{t} / R T\right) \exp \left(-\Delta G_{a} / R T\right)$

The proportionality factor that makes this inequality an equality depends on the collision frequency. The nucleation process is also dynamic, so that some molecules or ions will detach from the crystal and not all collisions will result in sticking. Including these additional processes complicates the mathematics but does not change the basic physical conclusion that there is a crtitical radius for homophase fluctuations in a solution; fluctuations that are larger than this critical radius will spontaneously grow and become nuclei for particle growth.

Equation (17) gives rise to an important and intuitive conclusion. Recall that $\Delta G_{v}$ is • negative for a spontaneous process. Thus, as the degree of supersaturation increases, $\Delta G_{v}$ 
becomes more negative and $r_{c}$ decreases. That is, as a solution becomes supersaturated to a greater degree, smaller fluctuations can produce critical nuclei and particle growth, and then it is easier to get spontaneous nucleation and precipitation.

Our derivation of the critical radius, Equation (17), was for a spherical geometry. If the cluster formed is in the shape of a cube of length $L$, then

$\Delta \mathrm{G}_{\mathrm{t}}=\mathrm{L}^{3} \Delta \mathrm{G}_{\mathrm{v}}+6 \mathrm{~L}^{2} \delta$

and

$\mathrm{L}_{c}=-\left(4 \delta / \Delta \mathrm{G}_{\mathrm{v}}\right)$

where $L_{c}$ is the critical length of the cube. For other geometries, e.g., needles or plates, $r_{c}$ can deviate from the spherical case [given by Equation (17)] by even greater amounts than for the case of a cube. See Nielson (1964), Kirkpatrick (1981), and Tadros (1987) for more details.

\subsection{Heterogeneous Nucleation}

It is also possible to nucleate supersaturated solutions without achieving spontaneous homogeneous nucleation. For example, in natural waters there is frequently sufficient colloidal iron hydroxide to act as nucleation sites for crystal growth of other compounds or sparingly soluble salts. The obvious result would be a particle of the precipitating phase with a central core of iron hydroxide. In a laboratory situation, this is less likely to occur since water is generally purified prior to its use.

Solid surfaces can also be the site of nucleation for supersaturated solutions (Nielsen, 1964). This is especially true if the surface is rough. A common technique in inducing crystallization in glass containers is to scratch the surface with a glass rod to provide the necessary roughness. Crystallization can sometimes be so rapid that the resulting crystalline masses form "rosettes" or needles that break apart or become detached and thus produce secondary sites for crystallization.

In general, when sites for heterogeneous nucleation are present, nucleation will occur at lower degrees of supersaturation than required for spontaneous homogeneous nucleation.

\subsection{Seeding Crystal Growth}

It is also possible to induce crystal growth by adding a small seed crystal of the desired type. For example, $\mathrm{Na}_{2} \mathrm{SO}_{4} \cdot 10 \mathrm{H}_{2} \mathrm{O}$ is the stable hydrate of $\mathrm{Na}_{2} \mathrm{SO}_{4}$ at room temperature. Thus, seeding a supersaturated aqueous $\mathrm{Na}_{2} \mathrm{SO}_{4}$ solution with one or more $\mathrm{Na}_{2} \mathrm{SO}_{4} \cdot 10 \mathrm{H}_{2} \mathrm{O}$ crystals would initiate crystallization of the thermodynamically stable hydrate. It is also possible to 
start the seeding at low degrees of supersaturation, and then grow crystals by gradually cooling the solution to reduce the solubility of the solute. This cooling technique can also be used to grow single crystals of a compound or salt from its melt.

A great advantage of these seeding techniques is that very large single crystals can be grown in some cases, in contrast to spontaneous homogeneous nucleation, which generally produces a larger number of smaller crystals.

In addition, by using seed crystals of a metastable form of the same substance or of a different substance that has a different crystal habitat (e.g., orthorhombic versus cubic), it is sometimes possible to induce precipitation of a solute differing in structure or composition from the normal precipitating phase.

\section{Ostwald Ripening}

In 1897, Ostwald reported that small crystals in a fresh precipitate frequently recrystallized with time to produce a smaller number of larger crystals. Since the surface energy of a solid affects whether a small cluster redissolves or becomes a growing nucleus, we would expect the Ostwald Ripening to be affected by related considerations (Aagaard and Helgeson, 1982; Voorhees, 1985).

When embryonic nuclei form during spontaneous homogeneous nucleation, they will grow by addition of ions or molecules from the solution phase until the concentration of the dissolved parent species decreases to the normal solubility. Particles with radii less than $r_{c}$ should dissolve as shown in Section 3. Once these small crystals have dissolved, then the remaining larger crystals will be in dynamic equilibrium with the solution and will have equal rates of dissolution and precipitation. Thus they will remain constant in size if surface energies are small relative to $\Delta \mathrm{G}_{\mathrm{v}}$. This will only be true if the precipitated particles are of a fairly large size, and in most cases this will not be true for initial nuclei.

Consider two ensembles of particles of the same material that are equal in total weight but different in size and number. For simplicity, let all particles in each ensemble be identical in size and all be spherical in shape. Let there be $n_{1}$ particles containing $j_{1}$ monomer units in ensemble 1 and $n_{2}$ particles with $j_{2}$ monomer units in ensemble 2 . Then,

$\Delta \mathrm{G}_{1}=\mathrm{n}_{1}\left[(4 / 3) \pi \mathrm{r}_{1}^{3} \Delta \mathrm{G}_{\mathrm{v}}+4 \pi \mathrm{r}_{1}^{2} \delta\right]$

and

$$
\Delta G_{2}=n_{2}\left[(4 / 3) \pi r_{2}^{3} \Delta G_{v}+4 \pi r_{2}^{2} \delta\right]
$$


Since we have the same weight of each material, the total volume $V_{i}$ of particles in each of the ensembles must be identical. Thus,

$V_{i}=n_{1}(4 / 3) \pi r_{1}^{3}=n_{2}(4 / 3) \pi r_{2}{ }^{3}$

Now consider a process in which the larger particles dissolve and recrystallize as smaller particles. The Gibbs energy change for this process is

$$
\begin{aligned}
\Delta G_{1}-\Delta G_{2} & =n_{1}\left(4 \pi r_{1}{ }^{2}\right) \delta-n_{2}\left(4 \pi r_{2}{ }^{2}\right) \delta \\
& =\left(4 \pi r_{1}{ }^{2} n_{1}-4 \pi r_{2}{ }^{2} n_{2}\right) \delta
\end{aligned}
$$

since the $\Delta G_{v}$ terms cancel. Here, 1 denotes the smaller particles and 2 denotes the larger particles (i.e., $r_{1}<r_{2}$ ). However, $4 \pi r_{i}^{2} n_{i}$ is just the surface area of an individual particle $i$ times the number of particles of that type. Let $A_{i}$ denote this total surface area for the $i^{\text {th }}$ type particles. Then,

$\Delta G_{1}-\Delta G_{2}=\delta\left(A_{1}-A_{2}\right)$

Since we assumed the particles were all spheres and $r_{1}<r_{2}$, then $A_{1}>A_{2}$. Thus,

$\delta G=\Delta G_{1}-\Delta G_{2}>0$.

We know from equilibrium thermodynamics that a process with positive $\delta G$ will not occur spontaneously, so we conclude that larger particles will not dissolve and form smaller particles.

If we now make the opposite assumption that particle 1 is larger than particle 2 , the same type of calculation gives $\delta G<0$, so a process of recrystallization of smaller particles to form larger particles is thermodynamically favored. If we were to repeat this calculation with a different geometry assumed for the precipitates (e.g., cubes, plates, needles), we would come to exactly the same conclusion; that is, smaller particles will always grow to form larger particles. As the more soluble smaller particles grow, the solubility gradually decreases. As we have just seen, Ostwald Ripening is firmly grounded in classical thermodynamics.

No numerical values were assumed for $n_{i}$ and $r_{i}$ in the above derivation. The formation of any larger particle is favored over a smaller particle. We thus expect that any system that is allowed enough time to reach complete thermodynamic equilibrium would consist of a single large particle containing of all the precipitate. In practice, this does not occur since, when the particles become large enough that surface energy becomes a minor contribution to the total Gibbs energy, the "driving force" for crystal growth becomes so small 
that crystal growth practically ceases. In addition, under these conditions, small thermal fluctuations can create fluctuations in $\delta G$ larger than the surface energy term and "swamp out" the tendency for crystal growth.

Varshal et al. (1983) studied the solubility of solid $\mathrm{RuO}_{2} \cdot \mathrm{nH}_{2} \mathrm{O}$ produced by adding alkali to an acidified solution of $\mathrm{Ru}(\mathrm{IV})$. At a $\mathrm{pH}$ of 4.5 , for example, the initial solubility was about $2.9 \times 10^{-4}$ moles/liter, but it decreased to $1.2 \times 10^{-5}$ moles/liter after 105 days. Thus, aging of the solution and precipitate caused the solubility to drop by about a factor of 25 . As expected, the rate at which the solubility changed became less at longer times.

\section{The Ostwald Step Rule}

Equilibrium thermodynamics can be used to predict the thermodynamically favored product from a reaction; in our case, this would be what type of precipitate will form from a supersaturated solution. In fact, precipitates that are not expected to form from purely thermodynamic considerations are frequently encountered. In 1897, this observation was formulated by Ostwald as his step rule (Morse and Casey, 1988). This rule can be stated as:

If a reaction can result in several products, it is not the stablest state with the least amount of free energy (Gibbs energy) that is initially obtained, but the least stable one, lying nearest to the original state in free energy.

As rephrased by Goldsmith, " ... [the] most disordered phases should form from a random system ..."

In some simple cases, the thermodynamically stable product is produced directly. Crystalline sucrose precipitates from its supersaturated solutions, as does crystalline $\mathrm{NaCl}$ from its solutions. However, for more complicated crystalline structures, particularly those with complex chemical stoichiometry, a metastable and more disordered phase will usually form initially. For example, precipitation of Ru(IV) solutions with base should produce crystalline $\mathrm{RuO}_{2}$ if thermodynamic stability were the only controlling factor. In fact, this precipitation always gives amorphous $\mathrm{RuO}_{2} \cdot \mathrm{nH}_{2} \mathrm{O}$, with $\mathrm{n}$ about 2 ( $\mathrm{Rard}, 1985$ ). In this and many other cases, kinetic rather than thermodynamic factors dominate for the initial precipitation.

Thermodynamic considerations guarantee that sooner or later the initial phase will completely transform into the thermodynamically stable phase. However, thermodynamics alone does not determine how long this transformation will take. The formation of calcium apatites in sediments will be used as an example. Phases that form by precipitation and then aging of the precipitate, in increasing order of thermodynamic stability, are: nonstoichiometric amorphous calcium phosphate, "dicalcium phosphate dihydrate" 
$\mathrm{CaHPO}_{4} \cdot 2 \mathrm{H}_{2} \mathrm{O}$, anhydrous "dicalcium phosphate" $\mathrm{CaHPO}_{4}$, "octacalcium phosphate" $\mathrm{Ca}_{4} \mathrm{H}\left(\mathrm{PO}_{4}\right)_{3} \cdot 2.5 \mathrm{H}_{2} \mathrm{O}$, tricalcium phosphate $\mathrm{Ca}_{3}\left(\mathrm{PO}_{4}\right)_{2}$, and finally hydroxyapatite $\mathrm{Ca}_{5}\left(\mathrm{PO}_{4}\right)_{3}(\mathrm{OH})$ or dahllite $\mathrm{Ca}_{10}\left(\mathrm{PO}_{4}\right)_{6}(\mathrm{OH})_{2}$. It has been reported that 10 years are required to transform the initial amorphous precipitates into "marine apatite" (Morse and Casey, 1988).

There are cases where the transformation of the initial metastable precipitate transforms into the thermodynamically stable phase in minutes to days. However, geological specimens of metastable minerals many millions of years of age are known, which is the opposite extreme. For example, coarse-grained hematite $\left(\alpha-\mathrm{Fe}_{2} \mathrm{O}_{3}\right)$ should transform to coarse-grained geothite $(\alpha-\mathrm{FeOOH})$ on thermodynamic grounds, but hematite has persisted in some sediments for hundreds of millions of years (Morse and Casey, 1988).

The rationale behind precipitation of metastable phases is related to the formation of embrionic nuclei from homophase fluctuations. It is easier for embryonic nuclei with a simple or disordered structure to form than nuclei for a chemically complicated crystalline phase. Once these nuclei form, they will give rise to precipitation of that metastable phase.

\section{The Implication of Ostwald Ripening and the Ostwald Step Rule for Production of Doped Phases}

It is sometimes desirable to produce co-precipitated or doped phases. For example, coprecipitation is sometimes used to remove small amounts of a radioactive element using larger amounts of a carrier (scavenger) phase. In other cases, the opposite may be desired; that is, recrystallization of material is used to reduce the concentration of impurities in an initial solid phase.

Consider the case of a minor contaminant cation $\mathrm{M}^{1}$ that can be co-precipitated into a bulk phase $M X_{n}$ to form $M\left(M^{1}\right) X_{n}$. For the case of thermodynamic equilibrium, the concentration of $\mathrm{M}^{1}$ in the solid phase will be proportional to its concentration in the solution phase (or to $\mathrm{C}^{\mathrm{x}+\mathrm{y}}$ if the solute dissociates) and the concentrations are related by a distribution coefficient $D$. This $D$ is defined as the ratio of the solute concentration (or $C^{x+y}$ ) in the solid phase to that in the solution phase.

If $\mathrm{M}^{1}$ is somewhat different than $\mathrm{M}$ in some of its properties (such as a larger size ion or an ion of different charge), then it will not "fit well" into the structure of the bulk phase and $D$ will typically be less than unity. However, if we produce $M\left(M^{1}\right) X_{n}$ by rapid precipitation, then we expect that the ratio of $\mathrm{M}^{1} / \mathrm{M}$ in the solid precipitate would be nearly identical to its ratio in the initial solution phase. This phase would be more disordered than pure $M X_{n}$, as expected from the Ostwald Step Rule. 
For our example just given, the initial metastable precipitate contains more $M^{1}$ than should be present at thermodynamic equilibrium. This precipitate will then "evolve" by the process of Ostwald Ripening. Recrystallization by Ostwald Ripening will be much slower than the initial nucleation and precipitation. It will occur slowly enough that material that recrystallizes will have a lower $\mathrm{M}^{1} / \mathrm{M}$ ratio, more characteristic of thermodynamic equilibrium rather than that of the initial metastable phase.

If $M^{1}$ has a different charge than $M$, then substitution of $M^{1}$ into $M X_{n}$ will require some type of charge and structural compensation from the host material. This will produce some type of defect structure. Two different types of ions could simultaneously codope into a crystal to achieve charge compensation. For example, $\mathrm{Cr}^{3+}$ and $\mathrm{Na}^{+}$together might replace two $\mathrm{Pb}^{2+}$ ions in some particular crystalline phase such as $\mathrm{PbSO}_{4}$, or two $\mathrm{Cr}^{3+}$ might replace three $\mathrm{Pb}^{2+}$.

If pure materials are desired, then allowing crystals to grow by Ostwald Ripening can produce the desired effect with time. However, if a doped material is desired, then it is necessary to separate the metastable solid phase from the solution phase without much delay. Otherwise, the resulting aged precipitates will contain a small core of more highly doped material surrounded by a layer of depleted material.

\section{Diffusion and Surface Reaction Controlled Growth}

We have not given any details of the growth mechanisms of particles during Ostwald Ripening. We have, however, discussed the step rule in which chemical reactions occur during evolution of precipitates with time. Typically, chemical reactions will occur predominantly at the surface of the particle, and thus reaction kinetics at the surface determine the particle growth rate during Ostwald Ripening for this case.

If a particle grows simply by Ostwald Ripening, then a depleted layer of solution will occur in the vicinity of the growing particle. This produces a concentration difference between the surface region of the particle and the bulk solution. Mass transport of dissolved solute will then occur to the depleted region by means of diffusion. When this becomes the dominant growth mode, particle growth is said to be diffusion controlled. Diffusion coefficients are typically about (1-2) $\times 10^{-5} \mathrm{~cm}^{2} / \mathrm{s}$ for aqueous ions; they are about half that size for bulkier molecules such as sucrose and are about $10^{-6} \mathrm{~cm}^{2} / \mathrm{s}$ for macromolecules such as proteins.

Transformation of amorphous hydrous oxides, silicate minerals, etc. into more thermodynamically favored species can involve surface absorption of $\mathrm{H}^{+}$or exchange of this hydrated $\mathrm{H}^{+}$with an alkali metal cation (e.g., reaction of an alkali metal feldspar with water). 
These surface reactions are generally much slower than diffusion and can be the kinetically limiting step in Ostwald Ripening.

More sophisticated analyses of Ostwald Ripening indicate that the distribution of grain sizes for surface reaction controlled crystal growth is symmetrical in the mean particle radius when scaled to the critical radius but, for the diffusion-controlled case, this distribution is skewed in favor of larger radii. In addition, the number of particles present are predicted to decrease as the inverse of time. Also, the mean radius of these particles increases as $t^{1 / 3}$ where $t$ is time. This last condition indicates that particle growth should be faster at shorter times and slower at longer times (Morse and Casey, 1988; Voorhees, 1985).

Occasionally dendritic crystals are produced, in which the crystals form as complicated branched structures. Metals are particularly prone to form such structures. Simple systems like $\mathrm{NaCl}$ form dendrites from their melt at $800^{\circ} \mathrm{C}$, but $\mathrm{NaCl}$ forms the normal faceted crystal when grown from aqueous solutions. The author has found that when aqueous rare earth perchlorate solutions are concentrated in a desiccator, crystallization occurs to form a glassy looking sheet. However, if saturated rare earth perchlorate solutions without an initial solid phase are cooled, then dendritic crystals frequently form instead.

A detailed discussion of dendritic growth is beyond the scope of this report. However, the cause of dendritic growth appears to be diffusion-induced instabilities at the solutionsolid interface (Doherty, 1980).

\section{Coagulation (Agglomeration) of Precipitates}

Any solid surface in contact with an electrolyte solution may become positively or negatively charged owing to preferential absorption of one type of ion on the surface. For solids of macroscopic dimensions, this charged layer has little affect on properties since bulk phase terms usually dominate. However, initial crystallization nuclei and colloidal material have a very high surface area-to-volume ratio. Since the amount of absorbed ions on a solid should be directly proportional to surface area, very small particles will have a very high surface charge-to-mass ratio compared to macroscopic particles. This will have a major effect on determining the stability of colloidal solutions.

Suppose a colloidal solid of composition $\mathrm{MX}_{\mathrm{n}}$ absorbs cations preferentially on its surface. These can be excess cations of type $M$ or some other type of cation $M^{1}$ (or mixtures of them) on the surfaces of the particles. The solution as a whole will be electrically neutral and will resist separation of charges to any major degree. Thus, the presence of the surface charge will induce the formation of a counter-ion layer of equal and opposite charge in the solution phase immediately adjacent to the particle's surface. 
Ions of a type not absorbed directly on the surface (so-called indifferent ions) can make up this outer charged layer. These counter ions will be more concentrated near the particle surface, and their concentration will "tail off" with increasing distance from that surface. This kind of distribution of ions is known as a diffuse double layer. The distance at which this diffuse double layer effectively ends is frequently termed the outer Helmholtz plane (OHP).

In addition to this charged double layer, van der Waals type attractive forces will be present, but are of shorter range. These attractive forces are of three kinds: dipole-dipole interactions, dipole-induced dipole interactions, and interactions based on fluctuations in the electron density distributions of the ions and molecules on and near the surfaces. They affect the Gibbs energy approximately as $r^{-6}$. See the book by Tadros (1987) for details.

The double layer thickness will depend on the concentration of the electrolyte in the solution phase. Increasing the electrolyte concentration compresses the double layer thickness, whereas decreasing the electrolyte concentration increases the double layer thickness. The range of this double layer is generally equated to the Debye length $\left(1.8 \times 10^{-10} / \sqrt{\mathrm{I}}\right)$, where $\mathrm{I}$ is the ionic strength and distances are in meters (Israelachvili and Adams, 1978; Osipow, 1962; Tadros, 1987). For distances in angstroms, leave off the power of ten.

Generally, attractive (van der Waals type) forces predominate up to about $(2-5) \times 10^{-10}$ meters from the particle surface, and repulsive forces dominate at slightly larger distances (Israelachvili and Adams, 1978; Osipow, 1962; Tadros, 1987; Yariv and Cross, 1979).

A third type of force has been described by Israelachvili and Adams (1978) from measurements of the interaction force between smooth mica surfaces. The magnitude of this repulsive force decays away roughly exponentially with distance.

Solutions of colloids and small particles sometimes clump together to form an aggregate particle that can be large enough to precipitate. This agglomeration process is known as coagulation or flocculation of precipitates.

For agglomeration to occur, the particles must first approach each other close enough. However, the counter ion layers near the surfaces of colloidal particles of the same type will have the same charge sign, and thus these particles will avoid each other due to electrical repulsion. Smaller colloidal particles, owing to their higher surface charge-to-mass ratio, will repel each other more strongly than larger colloidal particles. The repulsion between these particles decreases roughly as an exponential function of particle separation.

The concentration of electrolyte in the solution phase has a direct effect on the width of the charged double layer; higher electrolyte concentrations reduce the thickness of the 
double layer, whereas low electrolyte concentrations increase the thickness. Thus, high electrolyte concentrations allow the colloidal particles to approach each other more closely.

As mentioned briefly above, attractive (van der Waals type) forces also exist between colloidal particles, but they are of shorter range compared to the repulsion between counter ion layers on the charged particles. As the concentration of an added electrolyte is increased, the charged double layers are compressed so colloidal particles can approach each other more closely. Sufficiently high electrolyte concentrations can help make the colloidal particles approach close enough that the short range attractive forces then dominate and agglomeration can occur, giving rise to precipitation of coagulated particles. It has been found empirically that electrolytes with higher charges promote coagulation more effectively than electrolytes with lower charges. Yariv and Cross (1979) tabulated the critical concentrations of various electrolytes that cause coagulation of $\mathrm{Fe}_{2} \mathrm{O}_{3}$ and $\mathrm{Al}_{2} \mathrm{O}_{3}$ dispersions.

If reducing the thickness of a charged double layer promotes coagulation, then increasing its thickness should have the opposite effect. Washing a precipitate with water sometimes does just that; this process is known as peptization.

It should be noted that heating a solution disrupts the charged double layer structure on colloidal particles; this heating increases the Brownian motion of particles, and thus gives rise to more frequent and energetic conclusions. This will produce an enhanced rate of coagulation.

If a system is comprised of a suspension of spherical particles of uniform size (i.e., it is monodisperse) and if no interaction occurs between these particles until they come into direct contact and all contacts result in particles sticking together, then the rate of decrease in the total number of particles with time is given by

$1 / N_{t}=1 / N_{o}+k^{1} t$

Here, $N_{o}$ is the initial number of individual particles and $N_{t}$ is the number of particles at time t. Smoulchowski showed that, for rapid flocculation,

$\mathrm{k}^{1}=8 \pi \mathrm{Dr}$

where $\mathrm{D}$ is the diffusion coefficient of the particles and $\mathrm{r}$ is the particle radius. Typically, systems approximately follow Equation (28) during the early stages of rapid flocculation (Parfitt and Peacock, 1978).

If repulsive forces are important at a finite separation of particles, then there is a retardation of the rate of flocculation as given by Equations (28) and (29); typically this retardation (now known as slow flocculation) is one or two orders of magnitude from the 
predicted rate. In contrast, if attractive forces are more important than repulsive forces at finite separations between particles, then flocculation can occur slightly faster than predicted by Equations (28) and (29).

\section{Stabilization of Colloids}

Colloidal dispersions can sometimes be stabilized for extended periods of time given the proper conditions. This is sometimes desirable, for example, for the dispersion of pigments in inks and of both pigments and fillers in paints. Several types of stabilizers are described in Sections 9.1 and 9.2.

\subsection{Electrostatic Stabilization of Colloids}

As mentioned previously, many inorganic colloids have electrical charges on their surfaces due to ions in their crystal structure, and these surface ions give rise to the formation of a charged double layer in the region near the colloid. Since adding electrolytes to the solution phase compresses this double layer, their concentration can be adjusted to counterbalance the tendency to flocculate. Under this condition, the colloidal suspension is stabilized and shows no tendency to precipitate. In some organic systems, functional groups might be present on the surface of the colloid (e.g., carboxylate, amine, sulfonate), which can ionize and give rise to a charged double layer.

In other cases, such as some organic polymers, ionized groups are not present on the surface of the particles. However, it is sometimes possible to find compounds with ionizable groups that will physically absorb on the surface of the polymer, which then can produce a charged double layer. These can then be stabilized by adjusting the electrolyte concentration in the solution phase (Tadros, 1987).

\subsection{Steric Stabilization of Colloids}

It is sometimes possible to add an organic material to the solution that will cover the colloidal particles sufficiently well so that electrostatic forces are reduced enough to prevent flocculation. To work properly, the stabilizer must not only absorb on the colloid to form a thick and complete coverage, but the stabilizer must also be soluble in the solution phase. This type of stabilization is known as steric stabilization (Tadros, 1987).

Examples of materials that are commonly used as steric stabilizers are gelatin, egg white, vegetable oils, and (more recently) acrylic polymers. 


\section{Acknowledgments}

The author thanks Joan Schuyler for the word processing of this manuscript and Saundra Wander for the editing. 


\section{References}

Aagaard, P., and H. C. Helgeson, "Thermodynamic and Kinetic Constraints on Reaction Rates Among Minerals and Aqueous Solutions. I. Theoretical Considerations," American Journal of Science, 284, 237-285 (1982).

Doherty, R. D., "Dendritic Growth," in "Crystal Growth," B. R. Pamplin, editor; Pergamon Press, Oxford, second edition, 1980; pp. 485-520.

Israelachvili, J. N., and G. E. Adams, "Measurement of Forces Between Two Mica Surfaces in Aqueous Electrolyte Solutions in the Range 0-100 nm," Journal of the Faraday Society, Faraday Transitions 1, 74, 975-1001 (1978).

Kirkpatrick, R. J., "Kinetics of Crystallization of Igneous Rocks," in "Kinetics of Geochemical Processes," A. C. Lasaga and R. J. Kirkpatrick, editors; Mineralogical Society of America, Chelsea, Michigan, 1981.

Morse, J. W., and W. H. Casey, "Ostwald Processes and Mineral Paragenesis in Sediments," American Journal of Science, 288, 537-560 (1988).

Nielsen, A. E., "Kinetics of Precipitation," Pergamon Press (distributed by MacMillan Company, New York) 1964.

Osipow, L. I., "Surface Chemistry-Theory and Industrial Applications," Reinhold, New York, 1962.

Parfitt, G. D., and J. Peacock, "Stability of Colloidal Dispersions in Nonaqueous Media," in "Surface and Colloid Science," Vol. 10, E. Matijevic, editor, Plenum Press, New York, 1978.

Rard, J. A., "Chemistry and Thermodynamics of Ruthenium and Some of Its Inorganic Compounds and Aqueous Species," Chemical Reviews, 85, 1-39 (1985).

Tadros, Th. F., editor, "Solid/Liquid Dispersions," Academic Press, Harcourt Brace Javonovich, London, 1987.

Varshal, G. M., I. Ya. Koshcheeva, M. V. Akhmanova, and V. A. Dorofeeva, "Hydrolysis of Ruthenium(IV) in Perchlorate Solutions," Russian Journal of Analytical Chemistry, 38, 1395-1403 (1983).

Voorhees, P. W., "The Theory of Ostwald Ripening," Journal of Statistical Physics, 38, 231-252 (1985).

Yariv, S., and H. Cross, "Geochemistry of Colloid Systems-For Earth Scientists," Springer-Verlag, Berlin, 1979. 